EL CLIENTE COMO PROVEEDOR DE INNOVACIÓN; UN ENFOQUE DE CAPACIDADES DINÁMICAS EN PYME

\title{
EL CLIENTE COMO PROVEEDOR DE INNOVACIÓN. UN ENFOQUE DE CAPACIDADES DINÁMICAS EN PYME
}

\section{THE CUSTOMER AS AN INNOVATION PROVIDER. A DYNAMIC CAPABILITIES APPROACH IN PYME}

\author{
Juan Bernardino Arellano-Rodríguez*, José Sánchez Gutiérrez**, \\ Juan Mejía Trejo ${ }^{\star \star \star}$
}

\begin{abstract}
*Estudiante de doctorado. Centro Universitario de Ciencias Económico Administrativas, Universidad de Guadalajara Periférico Norte 799, Núcleo Universitario Los Belenes, Zapopan, Jalisco, 45100, México. Email: juanarellano1@hotmail.com

${ }^{\star \star}$ Estudiante de doctorado. Centro Universitario de Ciencias Económico Administrativas, Universidad de Guadalajara Periférico Norte 799 "G-306", Núcleo Universitario Los Belenes Zapopan, Jalisco, 45100, México. E-mail: jsanchez@cucea.udg.mx; jsanchez0202@hotmail.com

***Estudiante de doctorado. Centro Universitario de Ciencias Económico Administrativas, Universidad de Guadalajara Periférico Norte 799 "P-202", Núcleo Universitario Los Belenes, Zapopan, Jalisco, 45100, México. E-mail: jmejia@cucea.udg.mx; juanmejiatrejo@gmail.com
\end{abstract}

Dirección para recibir correspondencia: bernardino.arellano@academicos.udg.mx

Fecha de recibido: 14 de septiembre de 2017

Fecha de aceptación: 21 de febrero de 2018 
EL CLIENTE COMO PROVEEDOR DE INNOVACIÓN; UN ENFOQUE DE CAPACIDADES DINÁMICAS EN PYME

\section{RESUMEN}

La inversión en capital del conocimiento, y en innovación se considera por varios autores como elemento clave para la mejora en productividad. Este documento propone la utilización del enfoque de capacidades dinámicas para administrar el conocimiento, para la generación de innovación, lo cual llevaría a las empresas a mejoras en productividad, y a escalar posiciones en la cadena de valor global.

PALABRAS CLAVE: Administración del conocimiento del cliente. Innovación. Capacidades dinámicas.

\section{ABSTRACT}

The investment in knowledge and innovation is considered a key element for the improvement of productivity by some authors. In this document, the implementation of the dynamic capabilities approach is suggested, for the generation of innovation, which in return would lead to the improvement in productivity in companies in order to help increase positions in the global value chain.

KEY WORDS: management of customer's knowledge. Innovation. Dynamic capabilities.

\section{INTRODUCCIÓN}

Las micro, pequeñas, y medianas empresas en América Latina, tienen un nivel de productividad medio del $13 \%$, el $32 \%$ y el $43 \%$ con respecto a la productividad de las grandes empresas, lo cual y de acuerdo a la Organización para la Cooperación y el Desarrollo Económicos (OECD) y a la Comisión Económica para América Latina y el Caribe (ECLAC) (2012), es un porcentaje muy bajo en comparación con otras regiones del mundo.

En cuanto a México, se le considera como un país abierto, con una ubicación geográfica estratégicamente privilegiada, con bajos costos laborales, mano de obra cada vez más experta, ARELLANO-RODRÍGUEZ J. B., SÁNCHEZ GUTIÉRREZ J., MEJÍA-TREJO J. 
EL CLIENTE COMO PROVEEDOR DE INNOVACIÓN; UN ENFOQUE DE CAPACIDADES DINÁMICAS EN PYME

y prácticamente es ya un centro de manufactura mundial, por lo que de acuerdo a la experiencia tendría crecimiento en productividad al escalar en la cadena de valor, sin embargo, se considera que la participación de la PyME mexicana es muy baja en esa cadena de valor global, y se identifican entre las causas principales la brecha tan grande en capital basado en conocimiento y en innovación (OCDE, 2017), los cuáles son considerados como elementos claves para el incremento de productividad.

Como lo señalan Padilla \& Oddone (2016), la innovación es un elemento esencial en la transformación productiva de los eslabones de la cadena, tanto hacia mejores productos y servicios, como procesos o actividades de mayor valor agregado, que involucran el uso del conocimiento de una manera más intensiva.

Una de las grandes oportunidades para disminuir esa brecha en innovación es la administración del conocimiento del cliente. En este ensayo, se propone la manera en que las empresas pueden mejorar su posición en productividad y competitividad, al escalar en la cadena de valor, utilizando un enfoque de capacidades dinámicas para administrar el conocimiento del cliente hacia la generación de innovación.

\section{DISCUSIÓN}

De acuerdo a Teece, Pisano, \& Shuen (1997), en un entorno dinámico, cuando se presenta algún cambio económico u otro cambio drástico diferente, por ejemplo tecnológico, es muy probable que esas disrupciones o alteraciones conviertan en estratégicamente irrelevante $u$ obsoleto el recurso que actualmente representaba la ventaja competitiva, y por ello es que Teece, Pisano, \& Shuen (1997), proponen como opción para administrar estos cambios el enfoque de capacidades dinámicas, que se refieren a las habilidades de la empresa para integrar, construir, y reconfigurar sus competencias para administrar los entornos altamente dinámicos, y así no solamente adaptarse, sino como lo señalan Eisenhardt \& Martin, 2000; Teece, 2007, también configurar el cambio mismo de los mercados con innovaciones ya sea en productos, procesos, clientes, proveedores, canales de distribución.

Los entornos dinámicos tienen el cambio y el reto como constante, y Teece et al. (1997), Señalan que es precisamente ahí donde tiene su pertinencia la perspectiva de las capacidades dinámicas, las cuales permiten a la empresa enfocarse en su capacidad para crear nuevos recursos, renovarlos, o modificar/reconfigurar la mezcla de ellos, por lo que se vuelve crucial la ARELLANO-RODRÍGUEZ J. B., SÁNCHEZ GUTIÉRREZ J., MEJÍA-TREJO J. 
EL CLIENTE COMO PROVEEDOR DE INNOVACIÓN; UN ENFOQUE DE CAPACIDADES DINÁMICAS EN PYME

participación del equipo de la alta gerencia y sus creencias acerca de la evolución organizacional y su rol en el desarrollo de nuevas capacidades dinámicas (Rindova and Kotha 2001, p. 1274). Las capacidades dinámicas incluyen tres dimensiones (Teece et al. 1997; Teece, 2007; Barreto, 2010):

- Detección: consiste en escanear, buscar, explorar, oportunidades de mercados y tecnologías, tanto internamente, como con clientes, competidores, y proveedores actuales y potenciales, observando sus actividades de innovación, actualizándose en las fronteras del conocimiento, identificando las tendencias y mecanismos del desarrollo industrial (Teece 2000, 2007; O'Reilly and Tushman, 2008). Como lo mencionan Zhou, Zhou, Feng, \& Jiang (2017), las empresas capaces de desarrollar esta capacidad, tendrán una mejor comprensión de las necesidades de los clientes, para responder a ellas por la vía de innovación en canales de distribución, mercadotecnia, precios.

- Integración: Tiene que ver con la transferencia eficiente y efectiva de tecnología e información entre las diferentes áreas de la empresa, abriendo caminos al aprendizaje, compartir y transferir conocimiento y tecnología, información de mercados, internamente (Teece, 2007; Teece 2014; Cepeda \& Vera, 2007; Wang \& Ahmed, 2007).

- Reconfiguración: de acuerdo con Prieto, Revilla, y Rodríguez-Prado (2009), y Teece (2007), se refiere a la flexibilidad para reconfigurar las estructuras organizacionales, toma de decisiones en cada departamento, rediseño de procesos y procedimientos, rediseño de los mecanismos y redes de comunicación interna y externa, así como la disposición del conocimiento o recursos obsoletos.

El concepto de capacidades dinámicas soporta en gran medida el concepto de capital basado en conocimiento, que de acuerdo a OCDE (2017), tiene que ver con innovación de sistemas complejos, así como conocimientos gerenciales u organizacionales expertos, que permitan a la empresa detectar, integrar, y/o reconfigurar los recursos, capacidades, diseños, procesos, sistemas, o estructuras para lograr mejor desempeño, los cuáles se ubican entre uno de los cuatro tipos de capital basado en el conocimiento, llamado competencias económicas, incluyendo valor de marca, competencias tecnológicas y de gestión específica para las empresas, redes y estructuras organizacionales.

Acerca del concepto de innovación, el manual de Oslo (2005), ofrece la siguiente definición: 
EL CLIENTE COMO PROVEEDOR DE INNOVACIÓN; UN ENFOQUE DE CAPACIDADES DINÁMICAS EN PYME

"Una innovación tecnológica de producto, es la implementación/comercialización de un producto con características de desempeño mejorado así como como la entrega de servicios significativamente mejorados. Una innovación tecnológico de proceso es la implementación o adopción de un método nuevo o significativamente mejorado, para la producción o para la entrega; Puede involucrar cambios en el equipo, recursos humanos, métodos de trabajo, o combinaciones de estos elementos".

En este sentido, las empresas están en búsqueda permanente de recursos y capacidades que las habiliten hacia un nivel superior de desempeño, y una fuente muy importante de conocimiento e innovación para lograr mejoras en este rubro son las relaciones entre clienteproveedor (inter-firma), como lo mencionan Barnett \& Hansen (1996), a través de esos pequeños ajustes a las rutinas diarias que con el paso del tiempo actúan en la reconfiguración de los planes estratégicos formales, y se manifiestan a nivel de capacidades que se traducen luego en innovaciones, ya sea en el área de tecnología o de administración del conocimiento (Dyer \& Singh, 1998).

En relación a lo anterior, los clientes poseen información y conocimiento valioso para el proveedor, útiles para identificar maneras de como innovar, como crear valor, que les permita generar ventajas competitivas con ese cliente en particular o en mercados relacionados (Taherparvar, Esmaeilpour, \& Dostar, 2014), (Chesbrough 2006), y este enfoque de administrar el conocimiento del cliente convierte a ambos actores, cliente-proveedor, en co-creadores de innovación y de valor (Mejía-Trejo, J., Sanchez-Gutierrez, J., \& Haro-Beas, J. F. 2014)., pues el papel del cliente ya no se limita a la pasividad de ser un mero receptor del bien o servicio.

Las ideas y necesidades del cliente cuando se administran adecuadamente impactan fuertemente el futuro en innovación de la empresa (Rollins and Halinen, 2005; Zhang, Hoenig, Benedetto, Lancioni, Phatak, 2009) sobre todo en dos indicadores principales como son la velocidad de innovación y la calidad de la innovación (Liao, Wang, Chuang, and Shih, 2010; Lahiri, 2010).

De acuerdo a Cui \& Wu (2016), el conocimiento del cliente se debe considerar desde tres puntos de vista: Uno; Involucramiento del cliente como fuente de información, dos; involucramiento del cliente como co-desarrollador y tres, involucramiento del cliente como innovador; En cualquiera de estas tres etapas, ambas partes comparten experiencia y 
EL CLIENTE COMO PROVEEDOR DE INNOVACIÓN; UN ENFOQUE DE CAPACIDADES DINÁMICAS EN PYME

conocimiento, aprenden uno del otro y es la gran oportunidad para que el proveedor desarrolle esa capacidad especial llamada capacidad de absorción para obtener conocimiento externo, para administrarlo y desarrollarlo internamente (Du Plessis, 2007); (Taherparvar, N., Esmaeilpour, R., \& Dostar, M. 2014).

La mayoría de los modelos de innovación proponen su enfoque principalmente en innovación en procesos, productos, servicios, velocidad, y calidad (Chong, Chan, Ooi, \& Sim (2011); Wang and Wang, (2012); Mejía-Trejo et al. (2014); Fidel, Schlesinger \& Cervera (2015); Ju, Park, \& Kim (2016), y los analizan en función del desempeño de la firma.

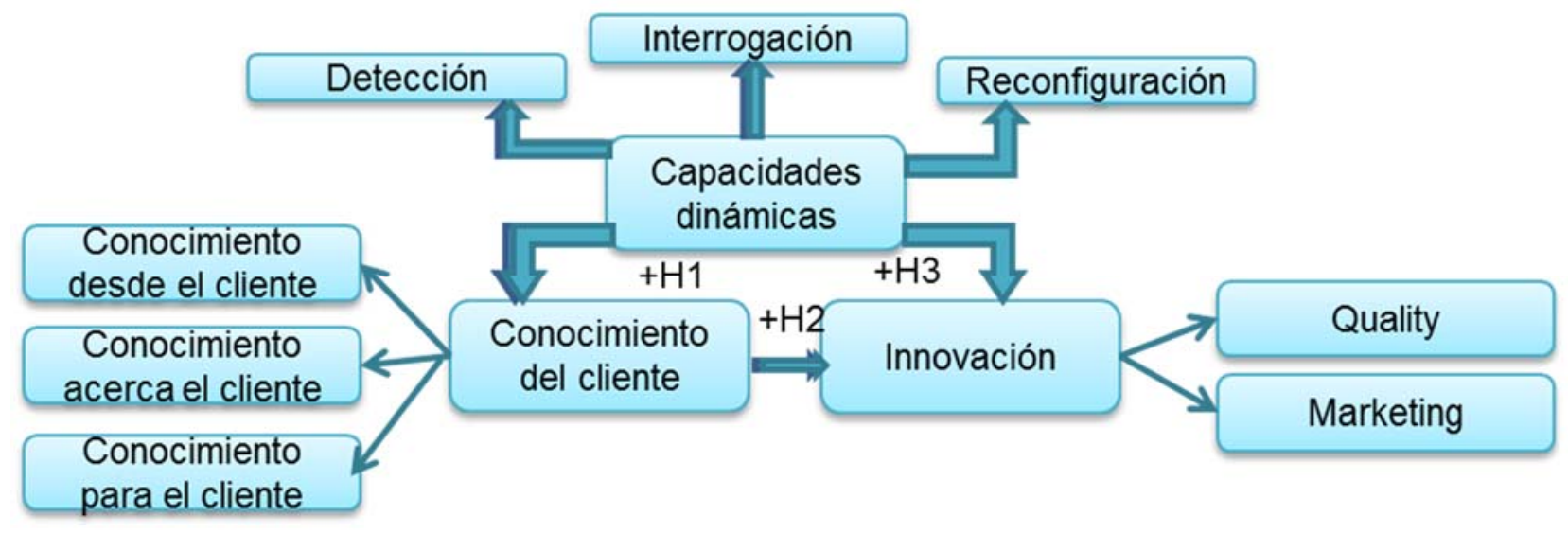

Figura 1. Modelo de la propuesta mencionada.

Fuente: Elaboración propia. Con base en revisión de literatura, 2017.

\section{CONCLUSIONES}

Este documento inicia con el análisis de la productividad de la PyME, señalada por la OCDE (2017) como muy baja y que la causa principal es el bajo nivel de innovación asociada con poca inversión en capital del conocimiento. Se revisaron enseguida las propuestas de varios autores respecto a la manera de administrar al papel de las capacidades dinámicas, la innovación, y el conocimiento del cliente como estrategias para incrementar la productividad y el desempeño de la firma. Al respecto, se incluyen enseguida algunos estudios empíricos relacionados y sus resultados: 
EL CLIENTE COMO PROVEEDOR DE INNOVACIÓN; UN ENFOQUE DE CAPACIDADES DINÁMICAS EN PYME

Existe evidencia empírica del impacto positivo que ejerce la administración del conocimiento del cliente en la innovación y en el desempeño de la firma (Mejía-Trejo et al. 2014), mediante estudios en empresas de Software en la Ciudad de Guadalajara, Jal.; por otro lado, también existe evidencia empírica acerca de la relación directa que tiene la orientación innovativa de la empresa con la manera de administrar el conocimiento del cliente, así como el impacto positivo que tiene la colaboración del cliente y la administración del conocimiento del cliente en los resultados de Marketing de la empresa (Fidel et al. 2015).

Por otra parte, existe evidencia empírica de la relación del CKM con varios factores de la empresa, como el impacto positivo de forma directa con el desempeño de negocios, mientras que también mostró impacto positivo indirecto con el incremento de la capacidad de innovación, a través del desempeño de negocios, e impacto positivo y directo en la capacidad de innovación; adicionalmente, reporta impacto positivo y directo de la capacidad de innovación en el desempeño de negocios (Taherparvar et al. 2014).

Con base en lo anterior se infiere que los beneficios en productividad, como lo señalan, Dyer \& Singh, 1998; son posibles en la cadena de valor cuando los socios invierten en relaciones específicas y combinan recursos en formas únicas, con intercambio importante de información, creación combinada de productos o servicios únicos, y sobre todo, dentro de los activos específicos inter-firma el concepto que le llaman especificidad de recursos, siempre y cuando se cumpla también con los requerimientos de un liderazgo y perfil de habilidades alineados para administrar la innovación.

\section{REFERENCIAS BIBLIOGRÁFICAS}

Barnett, W. P., \& Hansen, M. T. (1996). The red queen in organizational evolution. Strategic Management Journal, 17(S1), pp. 139-157.

Barreto, I. (2010). Dynamic capabilities: A review of past research and an agenda for the future. Journal of management, 36(1), pp. 256-280.

Chesbrough, H. W. (2006). The era of open innovation. Managing innovation and change, 127(3), pp. 34-41. 
EL CLIENTE COMO PROVEEDOR DE INNOVACIÓN; UN ENFOQUE DE CAPACIDADES DINÁMICAS EN PYME

Cepeda, G., \& Vera, D. (2007). Dynamic capabilities and operational capabilities: A knowledge management perspective. Journal of Business Research, 60(5), pp. 426-437.

Chong, A. Y., Chan, F. T., Ooi, K. B., \& Sim, J. J. (2011). Can Malaysian firms improve organizational/innovation performance via SCM?.Industrial Management \& Data Systems, 111(3), pp. 410-431.

Cui, A. S., \& Wu, F. (2016). Utilizing customer knowledge in innovation: antecedents and impact of customer involvement on new product performance. Journal of the Academy of Marketing Science, 44(4), pp. 516-538.

Du Plessis, M. (2007). The role of knowledge management in innovation. Journal of knowledge management, 11(4), pp. 20-29.

Dyer, J. H., \& Singh, H. (1998). The relational view: Cooperative strategy and sources of interorganizational competitive advantage. Academy of management review, 23(4), pp. 660-679.

Eisenhardt, K. M., \& Martin, J. A. (2000). Dynamic capabilities: what are they? Strategic management journal, 21(10-11), pp. 1105-1121.

Fidel, P., Schlesinger, W., \& Cervera, A. (2015). Collaborating to innovate: Effects on customer knowledge management and performance. Journal of business research, 68(7), pp. 1426-1428.

Ju, K. J., Park, B., \& Kim, T. (2016). Causal Relationship between Supply Chain Dynamic Capabilities, Technological Innovation, and Operational Performance. Management and Production Engineering Review, 7(4), pp. 6-15.

Lahiri, N. (2010), "Geographic distribution of R\&D activity: how does it affect innovation quality?" The Academy of Management Journal (AMJ), 53(5), pp. 1194-1209.

Liao, C.C., Wang, H.Y., Chuang, S.H. and Shih, M. (2010), "Enhancing knowledge management for R\&D innovation and firm performance: an integrative view", African Journal of Business Management, 4(14), pp. 3026-3038.

Mejía-Trejo, J., Sanchez-Gutiérrez, J., \& Haro-Beas, J. F. (Julio 2014). Customer Knowledge to Improve the Innovation: The Relationship in México. The 13th International conference of the Society for Global Business \& Economic development. Managing the "Intangibles": 
EL CLIENTE COMO PROVEEDOR DE INNOVACIÓN; UN ENFOQUE DE CAPACIDADES DINÁMICAS EN PYME

Business and Entrepreneurship Perspectives in a Global Context. Universitá Politecnica delle Marche. Ancona, Italy. Facoltá di Economia "Giorgio Fua", Piazzale Martelli, 8.

OCDE (2017), Estudios económicos de la OCDE: México 2017, OCDE Publishing, París. Recuperado de http://dx.doi.org/10.1787/9789264269040-es.

OECD/ECLAC (2012). Perspectivas económicas de América Latina 2013: Políticas de pymes para el cambio estructural, OECD Publishing, París. Recuperado de http://dx.doi.org/10.1787/leo-2013-es

Oslo Manual: guidelines for collecting and interpreting innovation data. OECD/ European Communities, 2005. Third Ed. http://www.oecd.org/science/inno/2367580.pdf

O'Reilly, C. A., \& Tushman, M. L. (2008). Ambidexterity as a dynamic capability:Resolving the innovator's dilemma. Research in organizational behavior, 28, pp. 185-206.

Padilla, R., \& Oddone, N. (2016). Manual para el fortalecimiento de cadenas de valor. Comisión Económica para América Latina y el Caribe (CEPAL).

Prieto, I. M., Revilla, E., \& Rodríguez-Prado, B. (2009). Building dynamic capabilities in product development: how do contextual antecedents matter? Scandinavian Journal of Management, 25(3), pp. 313-326.

Rindova, V. P., \& Kotha, S. (2001). Continuous "morphing": Competing through dynamic capabilities, form, and function. Academy of Management Journal, 44(6), pp. 1263- 1280.

Rollins, M. and Halinen, A. (2005), "Customer knowledge management competence: towards a theoretical framework", paper presented at The 38th Hawaii International Conference on System Sciences, Hawaii.

Taherparvar, N., Esmaeilpour, R., \& Dostar, M. (2014). Customer knowledge management, innovation capability and business performance: a case study of the banking industry. Journal of knowledge management, 18(3), pp. 591-610.

Teece, D. J. (2014). The foundations of enterprise performance: Dynamic and ordinary capabilities in an (economic) theory of firms. The Academy of Management Perspectives, 28(4), pp. 328-352. 
EL CLIENTE COMO PROVEEDOR DE INNOVACIÓN; UN ENFOQUE DE CAPACIDADES DINÁMICAS EN PYME

Teece, D. J. (2007). Explicating dynamic capabilities: the nature and microfoundations of (sustainable) enterprise performance. Strategic management journal, 28(13), pp. 13191350.

Teece, D. J., Pisano, G., \& Shuen, A. (1997). Dynamic capabilities and strategic management. Strategic management journal, pp. 509-533.

Wang, C. L., \& Ahmed, P. K. (2007). Dynamic capabilities: A review and research agenda. International journal of management reviews, 9(1), 31-51.

Zhang, J., Hoenig, S., Benedetto, A.D., Lancioni, R.A. and Phatak, A. (2009). "What contributes to the enhanced use of customer, competition and technology knowledge for product innovation performance? A survey of multinational industrial companies' subsidiaries operating in China", Industrial Marketing Management, 38(3), pp. 207-218.

Zhou, S. S., Zhou, A. J., Feng, J., \& Jiang, S. (2017). Dynamic capabilities and organizational performance: The mediating role of innovation. Journal of Management \& Organization, pp. 1-17.

Teece, D. J. (2007). Explicating dynamic capabilities: the nature and microfoundations of (sustainable) enterprise performance. Strategic Management Journal, 28(13), pp. 13191350.

Teece, D. J., Pisano, G., \& Shuen, A. (1997). Dynamic capabilities and strategic management. Strategic Management Journal, pp. 509-533.

Wang, C. L., \& Ahmed, P. K. (2007). Dynamic capabilities: A review and research agenda. International journal of management reviews, 9(1), pp. 31-51.

Zhang, J., Hoenig, S., Benedetto, A.D., Lancioni, R.A. and Phatak, A. (2009), "What contributes to the enhanced use of customer, competition and technology knowledge for product innovation performance? A survey of multinational industrial companies' subsidiaries operating in China". Industrial Marketing Management, 38(3), pp. 207-218.

Zhou, S. S., Zhou, A. J., Feng, J., \& Jiang, S. (2017). Dynamic capabilities and organizational performance: The mediating role of innovation. Journal of Management \& Organization, pp. 1-17. 